\title{
EFECTOS DEL CoVid-19 en los MeCanismos de CoORdinación E INTEGRACIÓN DE LA ARQUITECTURA ORGANIZACIONAL EN LAS EMPRESAS
}

\author{
José Feliz Marrero \\ Recibido: $15 / 7 / 2020 \bullet$ Aprobado: 20/9/2020
}

Cómo citar: Feliz Marrero, J. (2020). Efectos del Covid-19 en los mecanismos de coordinación e integración de la arquitectura organizacional en las empresas. Ciencia, Economía y Negocios, 4(2), 107-129. Doi: https:// doi.org/10.22206/ceyn.2020.v4i2.pp107-129

\begin{abstract}
Resumen
El presente artículo tiene como objetivo ofrecer un análisis del impacto que el entorno ocasionado por el COVID-19 está teniendo en los mecanismos de integración y coordinación interna de la arquitectura de las organizaciones. En adición, describe las caracteristicas distintivas que las empresas deben adoptar frente a este nuevo entorno. Se presenta un modelo integrador para el análisis de las arquitecturas organizacionales sustentando su explicación en los planteamientos presentados por diferentes teorias organizacionales, tales como contingencia estructural, estructuralista, dependencia de recursos, recursos y capacidades, situacional, de las relaciones humanas y del desarrollo organizacional. También expone las consideraciones esenciales sobre la relación entorno-estrategia-estructura. Enfatiza el articulo la necesidad de las empresas de adoptar cambios que articulen con sentido de oportunidad las estrategias, estructura, sistemas sociotécnicos, mecanismos de control, incentivos y factores cognitivos de las personas con el entorno, tomando estos elementos como determinantes del rendimiento organizacional. El artículo explica la necesidad de adoptar un cambio para que las empresas puedan sobrevivir bajo esta nuevas condiciones, generadas por el efecto COVID-19 y que creará las condiciones para el desarrollo de nuevos hábitos y costumbres en el trabajo, así como nuevos mecanismos de coordinación e integración de las unidades organizacionales y para la toma de decisiones efectivas. Estos elementos de la arquitectura de las organizaciones son factores determinantes para la sobrevivencia y el buen desempeño de las empresas bajo los efectos COVID -19.
\end{abstract}

Palabras clave: coordinación del trabajo; mecanismo de integración; arquitectura organizacional; empresa; gestión; cambio organizacional; COVID-19.

\footnotetext{
${ }^{a}$ Instituto Tecnológico de Santo Domingo (INTEC), Santo Domingo, República Dominicana. Correo-e: jose.feliz@intec.edu.do
}

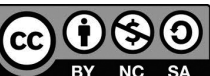

Esta obra está bajo licencia internacional Creative Commons Atribución-NoComercial-CompartirIgual 4.0 Internacional 


\title{
EFFECTS OF COVID-19 ON MECHANISMS OF COORDINATION AND INTEGRATION OF THE STRUCTURE OF THE ORGANIZATIONAL ARCHITECTURE OF COMPANIES
}

\author{
José Feliz Marrero
}

Received: 15/7/2020 Approved: 0/9/2020

\begin{abstract}
This article aims to analyze the impact of COVID-19 on the integration and coordination mechanisms of organizational architectures. I present a comprehensive model to analyze organizational architectures based on several organizational theories, including structural contingency, structuralism, resource dependence, resources and capacities, situational, human relations, and organizational development. This article emphasizes how organizations need to adopt changes that articulate with a sense of opportunity the strategies, the structure, the socio-technical systems, the control mechanisms, the incentives, and the cognitive factors of people with the environment. It also explains why companies are required to adopt changes to survive under the new conditions generated during the COVID-19 pandemic and how they can create the requirements for developing new working habits, coordination mechanisms, and integration of organizational units for effective decision making. These elements of the organization's architecture are essential factors for its survival and good performance under the COVID-19 pandemic.
\end{abstract}

Keywords: Work coordination; organizational architecture; integration mechanism; ompany; management; organizational change; COVID-19. 


\section{Introducción}

Las empresas, y por consiguiente las personas de los diferentes sectores productivos de bienes y servicios, enfrentan actualmente crisis en todos los ámbitos y dimensiones de su arquitectura organizacional. Una crisis compleja, llena de obstáculos y problemas que les dificultará operar de manera eficiente la época pos COVID, que sin lugar a dudas ha sido considerada una de las de mayor complejidad presentanda en los últimos 100 ańos, debido, básicamente, a que el sistema financiero global ha sido afectado y las economías de todos los países se encuentra en decrecimiento, atrapadas en un aumento del desempleo, quiebra y cierre de sus operaciones.

Para la Comisión Económica para América Latina y el Caribe (CEPAL) (2020) las economías han sufrido una caída, provocando un cierre de más de 2.6 millones de empresas y dejando a más de 200 millones de personas en la pobreza. Otras organizaciones, como el Banco Interamericano de Desarrollo (BID), el Fondo Monetario Internacional (FMI), la Organización para la Cooperación y el Desarrollo Económico (OCDE), la Organización Internacional del Trabajo (OIT), consideran que las políticas fiscales efectivas jugarán un importante rol en la estrategia global para encarar la situación y cambiar las condiciones de los sectores más frágiles, de manera que les permita crear mejores oportunidades para generar empleos y proteger a miles de trabajadores, que los han perdido. Adicionalmente, en los últimos cinco años, durante el período comprendido del 2014-2019, hubo un aumento de la deuda promedio de la región, alcanzando niveles cercanos al $50 \%$ del PIB (Suescun, 2020).

En este escenario, denominado proto-recesión por los expertos en economía, las empresas de cualquier sector, sin importar su tamaño y tipo de bienes y servicios que ofrecen sufren no solo el efecto de la crisis, sino el reto de sobrevivir ante la situación generada por una crisis en el sistema sanitario nacional, debido a que en la gran mayoría de los gobernantes han adoptados medidas de confinamiento para reducir el avance de la epidemia; lo que obliga a las empresas a reinventar sus modelos de negocios a través de la optimización de su arquitectura organizacional, en aras de que les permita replantear sus estrategias, potencializar sus 
capacidades, alinear sus procesos, rediseñar sus estructuras y estimular favorablemente los estados cognitivos de las personas para mejorar su desempeño y rendimiento.

Es relevante señalar que, bajo la situación actual, el ambiente y clima organizacional de las empresas deben garantizar las condiciones de confianza, seguridad y aprendizaje de sus colaboradores, para que puedan cumplir con sus propósitos con un alto sentido de responsabilidad, delegación y compromiso. Como es obvio, esto cambios generados impactarán en los modelos de gestión, los estilos y mecanismos de planificación, ejecución, control y seguimiento.

En esta nueva circunstancia, lo más importante a considerar es el factor humano, la creación de condiciones de trabajo que fomenten la creatividad e innovación, el énfasis en sembrar valores compartidos y en optimizar el poder de cambio y adaptación de las personas. Como consecuencia, se considera necesario rediseñar organizaciones que integren sistemas y procesos que faciliten el aprendizaje colaborativo y cuyas resultantes sean entregas de productos y servicios de alta calidad y valor para los clientes y la organización.

Para lograrlo, es necesario impulsar un proceso de cambio continuo, reflexivo, que conlleve la aplicación de un modelo de arquitectura organizacional integral, que considere la interacción de la tecnología, el ambiente y las personas como agentes esenciales de cambios.

Como se evidencia, el presente artículo intenta responder las siguientes interrogantes: jen qué medida los mecanismos de coordinación e integración de las estructuras organizacionales impactarán la arquitectura organizacional en las empresas por el efecto de COVID-19? ¿Cuáles serán las características distintivas que evidenciará la arquitectura organizacional de las empresas en este nuevo entorno?

\section{Coordinación e integración del trabajo}

Las entidades que agrupan personas y articulan de manera coordinadas los componentes de su sistema sociotécnico a través de la división del trabajo con propósitos, responsabilidades, autoridad jerarquizada y alcance delimitado, a los fines de lograr sus objetivos, metas y actividades establecidas son llamadas organizaciones (Schein, 1988). Estas metas 
y resultados alcanzados son una expresión natural de la especialización y la división del trabajo de manera coordinada, lo que significa que el proceso de asegurar el logro de las metas organizacionales demanda un esfuerzo importante de coordinación y articulación efectiva de las tareas.

Según Zerille (1978), las iniciativas orientadas a articular, balancear y estandarizar de manera armónica los subsistemas del sistema sociotécnico de las organizaciones es a lo que se le llama coordinación del trabajo. Esta coordinación se realiza tanto en las funciones formales de la empresa (organizar, dirigir, coordinar y controlar) como en sus mecanismos de interacción informal, lo cual conlleva mayores esfuerzos y dificultades para hacer efectiva la coordinación, demandando, por consiguiente, un alto nivel de confianza entre e inter colaboradores (Fukuyama, 2010).

Desde el punto de vista de la teoría estructuralista, las organizaciones coordinan e integran sus acciones con un enfoque racional, planificando sus actividades intencionalmente para el logro deliberado de sus metas y objetivos en contexto de procesos interdependientes. Según Blau y Scott (1962) citado por Bergoglio (2004), la teoría estructuralista analiza las empresas de manera integrada, tanto su dimensión formal como informal, profundizando en la estructura interna, concebida como un sistema social.

Según Etzioni (1994), la división del trabajo, responsabilidades y distribución del poder son características distintivas de las organizaciones y facilitan que las empresas realicen una mejor distribución de los recursos de los que disponen y un uso más eficiente. De acuerdo con Lawrence y Lorsh (1973), el incremento de la especialización del trabajo dificulta la coordinación del mismo, pues no se trata solamente de las diferentes especificaciones que tenemos para hacer el trabajo, sino que se expresa de la manera en que nos relacionamos y contribuimos en función al rol que ejercemos en el trabajo que realizamos.

De esa manera, tanto los procesos de reflexión colectiva como las estructuras organizacionales tendrán que coordinarse debido al impacto del COVID 19 en los procesos regulares de trabajo y en los instrumentos de normalización organizacional, generando cambios en sistemas de gestión, el seguimiento y supervisión del trabajo, redistribución de la tarea, mecanismos formales e informales de comunicación, entre otros (Hanzon, 1993). 
Estos cambios han generado una ruptura en su dinámica de coordinación, pues para superar y poder escalar a un nivel de coordinación efectiva del trabajo, se tendrán que redistribuir funciones, revisar normativas y definir con claridad los mecanismos de integración y coordinación de la estructura. En este proceso de regresar a una normalidad nueva, la resiliencia de los actores relevantes que conforma la estructura formal e informal de la organización juegan un rol esencial, pues son estos integrantes quienes adoptan el rol fundamental como entes de cambio.

Para Stoner (1995), la mejor manera de asegurar el alcance de las metas y objetivos organizacionales es logrando la cohesión interna de los departamentos y unidades funcionales, a través de un proceso de coordinación efectiva, comunicación clara de las tareas, flujo de información interdepartamental y fortalecimiento de los procesos de interdependientes, tanto de manera secuencial como recíproca.

De no ser así, se crean focos de dispersión de los compromisos y funciones que cada colaborador tiene asignado, creando las condiciones para el surgimiento de iniciativas e intereses particulares desviados de los compromisos establecidos entre la empresa y sus colaboradores.

Para Donnely, Gibson e Ivancevich (1995), la planificación se convierte en el instrumento que usan las organizaciones para lograr resultados individuales y de grupos de trabajo de manera coordinada. Por lo que ante un evento no planificado como lo es COVID-19, la acción inminente de las organizaciones ha sido reaccionar ante los hechos, provocando por consiguiente efectos desarticuladores en los mecanismos de coordinación e integración de la estructura.

La siguiente figura propone un modelo de análisis de la arquitectura organizacional que permite apreciar con mayor claridad el impacto del COVID-19 en la estructura y diseño de las organizaciones.

Según Hanzon (1993), el mecanismo que impulsa los procesos de reflexión colectiva y cohesión de las unidades empresariales es la organización informal, en cambio, la estructura formal es el eje central de los mecanismos de coordinación e integración de la estructura organizacional.

Por consiguiente, la estructura es la que crea las condiciones para que la empresa, mediante un proceso de planificación pueda coordinar efectivamente el trabajo, alcanzar sus aspiraciones y lograr las metas y objetivos propuestos (Hill, Jones y Schilling, 2015), lo que implica que las 
variables de estructuración: división del trabajo, departamentalización, jerarquía de decisiones y ámbito de control, son esenciales para que el trabajo pueda realizarse de manera integrada.

Como se observa en el cuadro 1, la base explicativa del modelo analítico para la arquitectura organizacional presentado en este artículo se sustenta en las siguientes teorías

- Teoría de contingencia estructural

- Teoría estructuralista

- Teoría de la dependencia de recursos

- Teoría de recursos y capacidades

- Teoría situacional o de contingencia

- Teoría de las relaciones humanas

- Teoría de desarrollo organizacional

Estas parten del contexto global para explicar la relación de la estrategia, la estructura, procesos, las personas y el rendimiento de las organizaciones.

\section{Entorno-Estrategia-Estructura}

La mejor descripción de la situación provocada por COVID-19 es la palabra contingencia, cuyas características y efectos en las empresas solo se puede explicar con la experiencia vivida y no necesariamente con el pensamiento racional. La correspondencia entre entono-estructuraestrategia se explica a través de la teoría situacional o de contingencia.

Es evidente que el panorama creado por la crisis sanitaria causada por el efecto del COVID-19 impacta considerablemente a la sociedad, a las organizaciones, personas, sus hábitos, cultura y, sobre todo, los entornos internos de las empresas, manifestándose de manera significativa en su desempeño y demandando un cambio importante en las personas, los procesos, la tecnología y cultura organizacional. De manera que la interacción ambiente-capacidades-estructura y desempeño, hay que asumirla desde una perspectiva holística, dentro de un contexto totalmente ecléctico, que favorece situar el diseño de la organización 
como centro de procesos de cambio y que impulsa la construcción de una nueva arquitectura organizacional que se exprese de una manera más inteligente, flexible y adaptativa a los procesos de cambio.

Como se observa en la figura 1 , el contexto global y empresarial impacta las estrategias, estructuras, procesos, personas y, consecuentemente, el desempeño de las organizaciones, generando una presión hacia un cambio favorable cuya consecuencia será un ajuste en todos los elementos de la arquitectura organizacional.

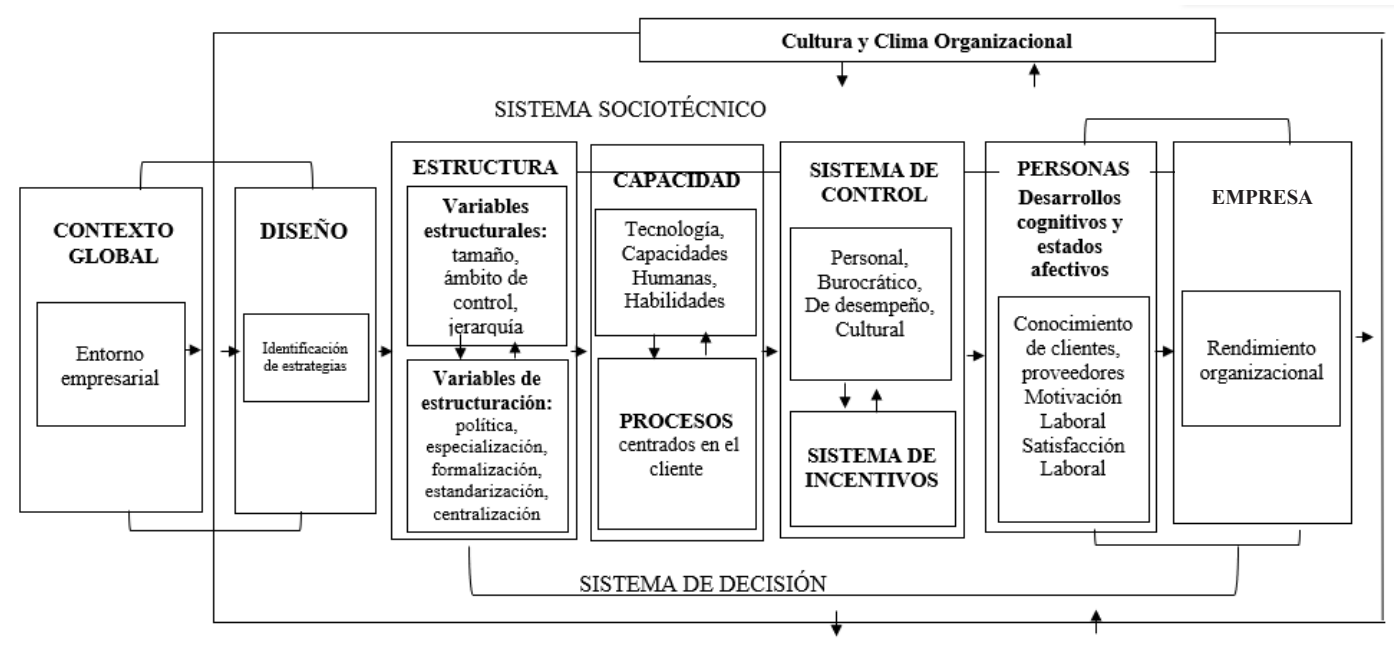

Figura 1. Modelo Analítico de la Arquitectura Organizacional

Fuente: elaboración propia 
Cuadro 1. Teorías oganizacionales que explican el modelo de análisis propuesto

\begin{tabular}{|c|c|c|c|}
\hline Teoría & Enfoque & $\begin{array}{l}\text { Exponentes de } \\
\text { las teorías }\end{array}$ & Periodo \\
\hline $\begin{array}{l}\text { Teoría de } \\
\text { contingencia } \\
\text { estructural }\end{array}$ & $\begin{array}{l}\text { Centra su enfoque en la } \\
\text { relación entorno externo y } \\
\text { como este afecta la estrategia, } \\
\text { estructuras y decisiones de la } \\
\text { empresa. }\end{array}$ & $\begin{array}{l}\text { Fred Fiedler, } \\
\text { Hersey y } \\
\text { Blanchard, } \\
\text { Vroom y Yetton } \\
\text { y Tannenbaum y } \\
\text { Schmidt }\end{array}$ & $\begin{array}{l}\text { Nace a finales de los } \\
\text { ańos cincuenta }\end{array}$ \\
\hline $\begin{array}{l}\text { Teoría } \\
\text { estructuralista }\end{array}$ & $\begin{array}{l}\text { Analizan las empresas en su } \\
\text { dimensión formal e informal } \\
\text { concebidas como sistema } \\
\text { social. Se enfocó en estudiar las } \\
\text { personas y el ambiente. Esta } \\
\text { teoría ve a la organización con } \\
\text { un enfoque de sistema, lo que } \\
\text { significa que un cambio en el } \\
\text { entorno genera un cambio en } \\
\text { los demás componentes del } \\
\text { sistema. }\end{array}$ & $\begin{array}{l}\text { Amitai Etzioni, } \\
\text { David Sills, } \\
\text { Burton, Clarke, } \\
\text { Jean Viet James } \\
\text { D. Thompson }\end{array}$ & $\begin{array}{l}\text { Desarrolladas en } \\
\text { la décadas del } \\
\text { cuarenta y del } \\
\text { cincuenta }\end{array}$ \\
\hline $\begin{array}{l}\text { Teoría de la } \\
\text { dependencia de } \\
\text { recursos }\end{array}$ & $\begin{array}{l}\text { Centra su atención en la } \\
\text { necesidad que tienen las } \\
\text { empresas de generar los } \\
\text { recursos que necesitan } \\
\text { para sobrevivir del } \\
\text { entorno, producto de la } \\
\text { interdependencia que se da } \\
\text { entre ellas. }\end{array}$ & $\begin{array}{l}\text { Jeffrey Pfeffer } \\
\text { y Gerald R. } \\
\text { Salancik } 1978\end{array}$ & Década de los 70 \\
\hline $\begin{array}{l}\text { Teoría de } \\
\text { recursos y } \\
\text { capacidades }\end{array}$ & $\begin{array}{l}\text { Explica la robustez y riesgo } \\
\text { interno a los que se exponen } \\
\text { las organizaciones y las } \\
\text { condiciones para potencializar } \\
\text { las oportunidades del entorno, } \\
\text { así como en la relevancia para } \\
\text { el desarrollo de competencias } \\
\text { y habilidades distintivas, } \\
\text { necesaria para enfrentar } \\
\text { un entorno cada vez más } \\
\text { turbulento y complejo }\end{array}$ & $\begin{array}{l}\text { Barney, J. (2001). } \\
\text { Brunner, J. } \\
\text { (2000). } \\
\text { Grant, R. (1996). }\end{array}$ & Década de los 90 \\
\hline
\end{tabular}




\begin{tabular}{|c|c|c|c|}
\hline Teoría & Enfoque & $\begin{array}{l}\text { Exponentes de } \\
\text { las teorías }\end{array}$ & Periodo \\
\hline $\begin{array}{l}\text { Teoría } \\
\text { situacional o de } \\
\text { contigencia }\end{array}$ & $\begin{array}{l}\text { Centran su trabajo entre la } \\
\text { relación ambiente-estructura } \\
\text { y los procesos de gestión } \\
\text { adminstrativa. Explica que } \\
\text { no hay una única y mejor } \\
\text { manera para gestionar los } \\
\text { procesos e individuos en las } \\
\text { organizaciones }\end{array}$ & $\begin{array}{l}\text { Chandler, } \\
\text { Skinner, Burns, y } \\
\text { P. Lawrence y J. } \\
\text { Lorsch }\end{array}$ & $\begin{array}{l}\text { Desarrollada en } \\
1972\end{array}$ \\
\hline $\begin{array}{l}\text { Teoría de las } \\
\text { relaciones } \\
\text { humanas }\end{array}$ & $\begin{array}{l}\text { Su enfoque está en las } \\
\text { personas y, sobre todo, en las } \\
\text { condiciones laborales sobre las } \\
\text { que desarollan su trabajo. Hace } \\
\text { énfasis en las personas y en las } \\
\text { organizaciones como equipos } \\
\text { de trabajo }\end{array}$ & $\begin{array}{l}\text { Desarrollada por } \\
\text { Thomas J. Peters } \\
\text { y Robert H. } \\
\text { Waterman }\end{array}$ & $\begin{array}{l}\text { Desarrollada en la } \\
\text { década de los } 30 .\end{array}$ \\
\hline $\begin{array}{l}\text { Teoría de } \\
\text { desarrollo } \\
\text { organizacional }\end{array}$ & $\begin{array}{l}\text { Centra su enfoque en la } \\
\text { persona y en el cambio } \\
\text { organizado dentro de un } \\
\text { contexto de sistemas abiertos }\end{array}$ & $\begin{array}{l}\text { Warren Bennis, } \\
\text { Edgar Shani, } \\
\text { Robert Blake y } \\
\text { Janet Mouton }\end{array}$ & $\begin{array}{l}\text { Desarrollada en la } \\
\text { década de los } 70\end{array}$ \\
\hline
\end{tabular}

Fuente: elaboración propia.

El enfoque sustentado en la teoría situacional o de contingencia de las organizaciones y cuyos precursores, según Rivas (2001), son Lawrence y Lorsch, Chandler, Burns Stalker y Woodward focalizan sus investigaciones en la relación ambiente-estructura y su impacto en los procesos y técnicas de gestión administrativas dentro de un contexto de sistema abierto. Sostienen que no existe un modelo organizacional único para alcanzar organizaciones eficaces, por lo que tampoco existe una única vía y modo de organizar la empresa para el logro de sus objetivos.

Según los planteamientos de Burns y Stalker (1961) citado por Donaldson (2001), las organizaciones cuyas características las tipifican como orgánicas, serán más adaptativas al nuevo entorno producido, en este caso por el efecto de la Pandemia, que aquellas denominadas mecánicas y cuya configuración están estructuradas para sistemas estables.

Es importante puntualizar que las estructuras de las organizaciones y su diseño, que hacen a las empresas más flexibles y con condiciones de 
responder de manera rápida, con sentido de urgencia y de oportunidad, se convierten en un requerimiento de sobrevivencia esencial para todas las entidades afectadas por los efectos del COVID-19. Esta situación obliga a que, bajo esta nueva realidad de cambios continuos, las empresas deben adoptar nuevos modelos mentales entre sus colaboradores, así como desaprender viejos patrones organizacionales, por ende, aprender nuevas formas de colaboración y gestión de sus procesos de trabajo.

Desde la perspectiva de la teoría de la contingencia estructural, Donaldson (2001) plantea que el entorno y la estrategia son variables contingentes determinantes de la estructura, influenciado por las características contextuales. Su valor agregado es hacer más eficientes los procesos y afectar de manera positiva los mecanismos de control, integración, jerarquía y la incertidumbre de tarea, producto del cambio que se experimenta en las demandas y aspiraciones de los clientes, presionados por sobrevivir ante esta nueva realidad.

Mintzberg (2013), considera la coordinación y mecanismo de integración como referentes importantes para el diseńo de la estructura. La adaptación mutua, supervisión directa y la normalización de procesos y resultados son los mecanismos de coordinación esenciales de las organizaciones (Mintzberg, 1993).

\section{a) Adaptación mutua}

Es el mecanismo de coordinación donde los individuos interactúan a través de las tareas que ejecutan mutuamente con una simple comunicación informal. En el contexto actual, este tipo de coordinación está muy limitado debido a la ruptura provocada por la pandemia en la rutina normal de operaciones, debido, principalmente, a que las empresas no estaban preparadas para un cese de las operaciones formales, afectando por consiguiente los procesos naturales que crean las condiciones para formentar los mecanismos de comunicación informal a lo interno de la empresa. De igual modo, muy pocas empresas cuentan con herramientas tecnológicas para controlar el trabajo remoto, lo que hace difícil su monitoreo y seguimiento, en adición a la falta de preparación de los empleados para manejarse en este ambiente. 


\section{b) Supervisión directa}

Este mecanismo de coordinación, que se manifiesta a través de la jerarquía de decisiones donde una persona lidera, ordena y controla las acciones de otras, expresándose a través de una conexión supervisor-supervisado y que se fundamenta en la cadena de mando: autoridad, responsabilidad y comunicación formal bajo el entorno COVID-19, se percibe poco controlado, ya sea por la falta de instrumentos tecnológicos que faciliten el monitoreo remoto del trabajo o por la falta de formación del supervisor para gestionar bajo este nuevo entorno de manera eficiente el trabajo de sus colaboradores a su mando.

\section{c) Normalización}

Los viejos instrumentos normativos, no han cambiado, ni han sido ajustados a esta nueva realidad, lo que indica que se requiere una actualización y normalización del diseño de los puestos de trabajo y de los procesos. Consecuentemente, la normalización de los resultados ha sido afectada, demandando ajustes en las metas e indicadores de desempeño de la empresa, creando las condiciones para el desarrollo de nuevas creencias, hábitos, costumbres y valores que caracterizarán la nueva cultura organizacional pos COVID-19.

Kast y Rosenzweig (1995), consideran la estructura como el modelo establecido de los vínculos entre las unidades organizativas de las empresas impactando el comportamiento organizacional de las mismas.

Por otra parte, Robbins (2010), asigna a la estructura de las organizaciones las mismas funciones que tiene el esqueleto en los seres humanos. Con atribuciones de facilitar la comunicación, coordinación del trabajo y articulación de los mecanismos que cohesionan las unidades de trabajo.

Para Chiavenato (2006), citando las investigaciones realizadas por Lawrence y Lorsch (1973) sobre el entorno de la empresa y su impacto en la estructura y los procesos administrativos, la diferenciación e integración representan los ejes críticos que son los problemas fundamentales de la empresa. 
Obviamente, que bajo los efectos de un entorno caracterizado por crisis sanitaria, financieras, operacional y emocional, y que ha generado cierre de empresas, distanciamiento de los colaboradores, cambio en la modalidad de interacción y modelos de gestión de las empresas, se ha producido, consecuentemente, una ruptura en los mecanismos de integración en cuanto la redistribución y división del trabajo.

En el ambiente COVID-19, el trabajo se realiza de manera remota en formato de teletrabajo y, en ocasiones, compartida con presencialidad mínima. Esta nueva realidad plantea el reto de repensar, bajo un nuevo contexto, una arquitectura organizacional diferente debido a la necesidad de rediseñar nuevos mecanismos de coordinación del trabajo a través del medio remoto o virtual. Para ello se deben crear todas las condiciones que permitan establecer y evidenciar entre los colaboradores y, principalmente, en la relación supervisor-supervisado niveles de confianzas superiores. Según la OIT (2020) la confianza es el "pegamento" que mueve la estructura. Sin la confianza y apoyo del liderazgo de la empresa hacia los colaboradores no hay vías posibles de éxito en la modalidad de trabajo remoto, pues es en este nivel gerencial donde se expresan los mayores dificultades para adoptar la nueva modalidad de trabajo pos COVID-19.

Mintzberg (1991), manifiesta que el diseño organizacional explica la forma como la organización funciona y coordina los procesos de trabajo, gestiona a las personas, asigna los niveles de autoridad y define los flujos de información y los mecanismos de toma de decisiones.

Se entiende que para que estos procesos sean efectivos, es fundamental contar con los recursos y capacidades organizacionales, lo que significa que las arquitectura organizacional de las empresas pos COVID-19 debe expresar de manera colectiva mecanismos innovadores de integración y coordinación, adoptar oportunamente y con sentido de urgencia el cambio, así como buscar vías distintas y creativas de solución de situaciones que den respuestas a los requerimientos de clientes dentro un ambiente de trabajo altamente positivo y motivador, y que sean oportunas, satisfactorias y superiores a las de los competidores. 


\section{Entorno-Recursos}

La teoría de la dependencia de recursos establece que debido a que las organizaciones no tienen los recursos y condiciones suficientes a lo interno para subsistir, tienen la necesidad del desarrollo de mecanismos de captación de recursos del entorno para asegurar su sobrevivencia y competitividad, al mismo tiempo, enfrentarse a situaciones de interdependencia como forma de querer controlar el entorno (Pfeffer, 1987). En tal sentido, esta teoría se enfoca en analizar los modos de coordinación que las empresas a lo interno y externo de ellas deben establecer para asegurar una menor dependencia de los recursos del entorno.

Según Hall (1996), la sobrevivencia organizacional implica que los directivos y ejecutivos de las empresas seleccionen estrategias efectivas que faciliten transar con los actores del entorno el intercambio de recursos como alternativa de sobrevivencia. Dentro de este mismo contexto y alcance de esta teoría, Peterson, (2011), señala que a lo interno de la arquitectura de las organizaciones y, principalmente, en el desarrollo de la estrategia y la estructura, hay agentes con influencia en la práctica organizacional que controlan los recursos indispensables que las empresas requieren, por lo que se hace necesario comprender que los procesos de adopción del cambio, que demanda el entorno a las organizaciones, es una condición esencial de sobrevivencia organizacional.

Como se puede observar en la figura 2, el control externo, que en condiciones normales es fuente de recursos para las empresas, hoy, bajo las condiciones de crisis creadas por la pandemia provocada por el COVID-19, genera incertidumbre y desbalance en función de la necesidad de captura de recursos externos y las condiciones de rupturas de los sistemas socioténicos, que demandan ajustes profundos en la arquitectura de las organizaciones. 


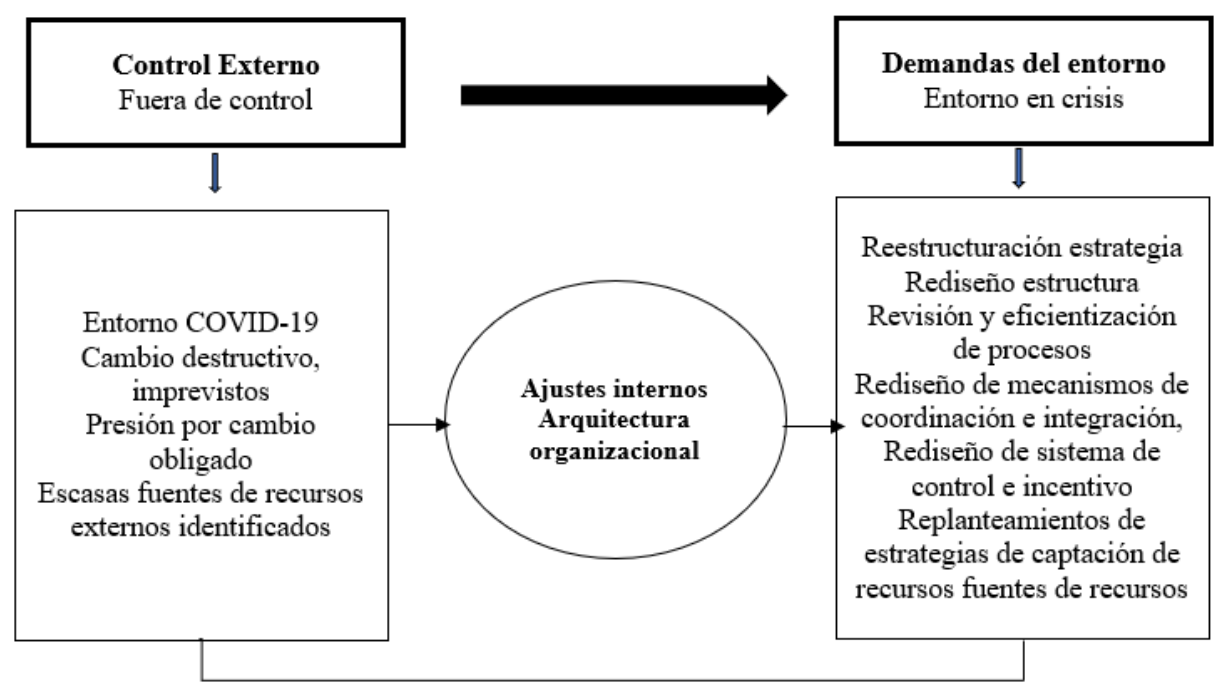

Figura 2. Influencia del contexto COVID-19 en la Arquitectura de las Organizaciones Fuente: adaptación basada en Pfeffer \& Salancik (1978) por José Feliz.

En esa misma idea, Gnyawali y Madhavan (2001) puntualizan que a través de los nexos de cooperación mutua que se forman por medio de las redes y que se generan en la dinámica que imponen los diseńos organizacionales, se crean condiciones que les permiten a los actores relevantes de las organizaciones asumir compromisos y compartir tareas y propósitos comunes, independientemente de las acciones que de manera particular cada colaborar debe asumir para mejorar las tareas asignadas o correspondientes a su puesto de trabajo.

En ese sentido, el análisis de los recursos y capacidades es considerado esencial para el entendimiento de la situación interna de la empresa, así como para la formulación de estrategias (Navas y Guerras, 2001). Pues, aunque centra su enfoque en los activos que la empresa controla y sus efectos en los resultados alcanzados (Wernerfelt, 1989), se establece como un factor importante que crea valor y permite a la empresa tener resultados superiores al promedio.

De manera que, para lograr niveles superiores de éxitos bajo estas condiciones, las organizaciones deben estar consciente de algunas condiciones que este nuevo entorno les crea, tales como: 
1. Cuentan con recursos limitados para satisfacer la demanda.

2. El recurso humano será el más valioso bajo esta condición de crisis, por lo que debe recibir de parte del liderazgo de la empresa especial atención.

3. La coordinación y cooperación serán acciones fundamentales para el éxito de las organizaciones.

4. El esfuerzo por articular los mecanismos de integración y coordinación del trabajo serán vitales para poder sobrevivir.

5. Las organizaciones tendrán que reinventar e innovar nuevas formas de trabajar, nuevos productos, servicios, mecanismos de relaciones y de manejo de la interdependencia con el entorno.

\section{Entorno-Persona}

Desde la perspectiva de las nuevas teorías de las relaciones humanas, se sustenta como elemento fundamental que la organización debe crear el ambiente para mejorar las condiciones laborales del trabajador, capacitándolo para manejar tareas complejas, integrando tecnología para simplificar el trabajo, creando condiciones de seguridad y un ecosistema de trabajo que permita y estimule la innovación y creatividad, así como necesario el establecimiento de sistemas de compensación y beneficios competitivos.

En consecuencia, la empresa en este entorno enfrenta nuevas condiciones de trabajo, impuestas por la situación provocada por la pandemia causada por el COVID-19. La arquitectura organizacional de los negocios será necesariamente el resultado de la interacción del entono, las estrategias, las capacidades organizacionales, la estructura, el estado cognitivo y el desempeño organizacional, como lo presenta la figura 1, generando por consiguiente las condiciones para el desarrollo de una nueva cultura organizacional.

De esta interacción y de la mayor comprensión y entendimiento de la situación de parte del liderazgo de la empresa, dependerán los logros y resultados alcanzados, así como los niveles de desempeño laboral obtenidos por parte de los colaboradores que integran la empresa. 
De ahí, se puede afirmar la interacción recíproca que se establece entre la cultura y la estructura formal de las empresas, así como de la coexistencia de ambas en organizaciones eficaces (Bierly y Spender 1995). Para Kopelman, Brief y Guzzo (1990) las prácticas de gestión humana, que caracterizan el contexto laboral y delimitan totalmente la cultura, y el clima son factores determinantes en el desempeño de las organizaciones. Sin embargo, en las condiciones actuales, el entorno es el determinante de los nuevos estilos de gestión, interacciones entre personas, adopción de nuevas prácticas de gestión humana y, por consiguiente, del establecimiento de una nueva cultura de interacción laboral que afecta el clima y redefine la cultura, impactando de manera directa en los resultados del negocio.

\section{Arquitectura Organizacional pos Covid-19}

Por consiguiente, se recomienda avanzar que las organizaciones pos COVID-19 se sustentarán en un modelo de interdependencia estratégica y estructural, pues la epidemia ha generado una ruptura de los sistemas sociotécnicos de las organizaciones, provocando, por consiguiente, un desbalance entre las expectativas y necesidades de los colaboradores y stakeholder con el modelo y dinámica del negocio, así como con sus resultados.

Es obvio que una ruptura de los sistemas sociotécnicos significa que los vínculos e intercambio entre las personas, las máquinas, los sistemas de trabajo y el ambiente laboral no actúan de manera cohesionada, significando un enfriamiento de las relaciones sociales y técnicas en las organizaciones y una desvinculación del accionar de la empresa con el ambiente y sus retos que, ante un nuevo entorno, les espera. (Cummings y Worley, 2007).

Según Trist (1981), los empleados mejoran su desempeño cuando se sienten empoderados y con mayor nivel de autonomía para tomar decisiones, por lo tanto, la arquitectura organizacional de las empresas pos COVID-19 está obligada a generar más autonomía y compromiso laboral, para garantizar el logro de los resultados óptimamente deseables. 
En adición, se creará la necesidad de generar normativas donde el teletrabajo o, mejor dicho, parte de los beneficios que la empresa se ahorra o puede ahorrarse del teletrabajo se transfieran a los empleados. Lo que significa que la organización pos COVID-19 debe ser una que genere un balance entre lo humano y el negocio. Las arquitecturas de este tipo de organización favorecen que las redes de apoyo virtuales ganen preponderancia y donde la situación dominante y centralizada eran fuentes tradicionales, se debiliten. En ese mismo sentido, las estructuras y los factores de gobernabilidad y de coordinación podrán fracturarse, sobre todo los relacionados a los mecanismos de adaptación mutua, normalización de los resultados de trabajos y supervisión directa.

Estos factores de coordinación actúan como un continuum, en cuanto a que para lograr los resultados esperados debe asegurarse cohesión en todos los niveles de coordinación e integración de los diseños y estructuras organizacionales.

Por esta razón, el gran reto de las empresas frente a esta crisis pos COVID-19 es un alto nivel de desarrollo y compromiso del liderazgo, capaz de impulsar las iniciativas que permitan y crean las condiciones para la conformación de una arquitectura que asegure alineación y coordinación efectiva de todos los mecanismos de integración y coordinación requerida para alcanzar resultados superiores al promedio del sector industrial al que pertenece la empresa.

Para nadie es un secreto que la organización pos COVID-19 requerirá ser adaptativa en su visión estratégica, inteligente en la articulación de sus procesos y flexible en su configuración estructural. Esto significa, que para lograr niveles de desempeño óptimos las organizaciones deberán configurar su arquitectura con un alto nivel de adaptabilidad y flexibilidad al cambio.

Por lo tanto, la arquitectura de las organizaciones pos COVID-19 se plantean como un reto articulador de la integración de los tres sistemas: de tarea, administrativo y social, que han sido afectados. (véase figura 3) 


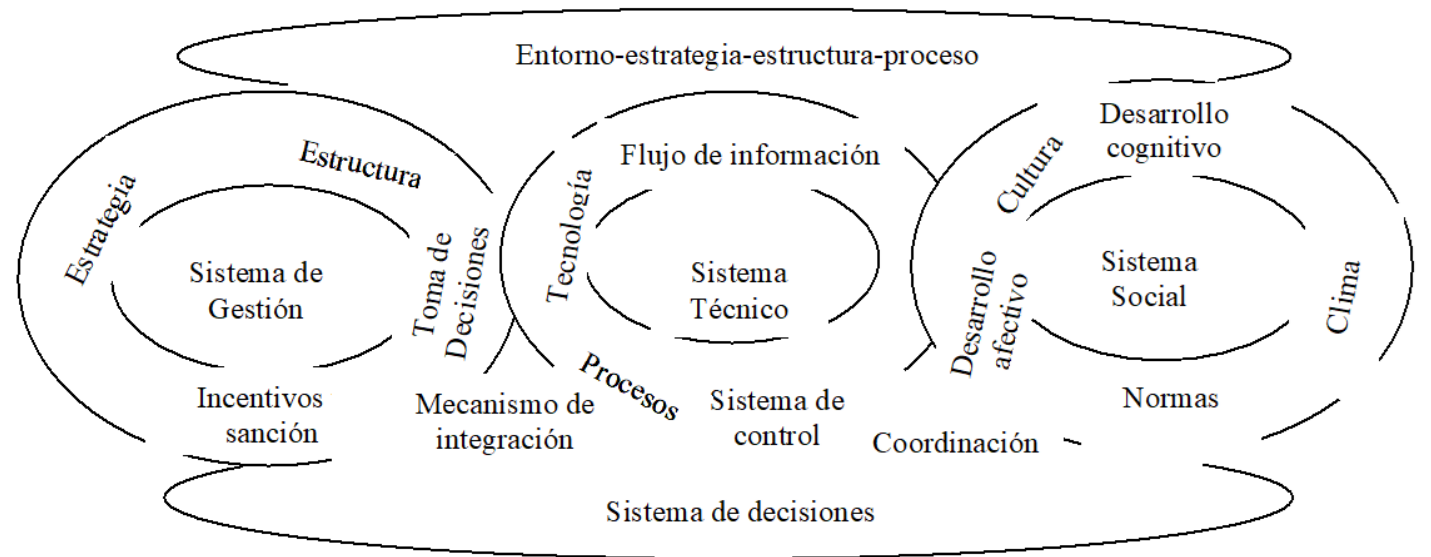

Figura 3. Interrelación recíproca de los componentes del sistema socio-técnico

Fuente: elaboración propia.

En el caso del subsistema de tarea o técnico los procesos, debe revisarse y adaptarse al trabajo remoto, compartido con el trabajo presencial, a los fines de asegurar un flujo de información, adecuación tecnológica y una revisión de las actividades requeridas para hacer el trabajo. Es necesario que estén en consonancia con el sistema social que involucra las normas, cultura, hábitos, reglamentos, políticas y los aspectos vinculados con el desarrollo cognitivo y afectivo de los colaboradores en la empresa.

Ahora bien, para que se pueda retomar la integración requerida resultado de la situación actual, las empresas deben revisar la interrelación recíproca que se da en el sistema sociotécnico, como se evidencia en la figura 3.

La inteligencia en la arquitectura organizacional está orientada a establecer una alineación efectiva entre los factores contingentes, capacidades y procesos, variables estructurales y de estructuración del diseño, el desarrollo cognitivo y estados afectivos de las personas con el desarrollo organizacional. Es necesario que para lograr altos rendimientos las organizaciones operen bajo un clima organizacional positivo. 
Contar con una arquitectura organizacional inteligente permite que las empresas sean más adaptativas a los procesos de cambios y estén en capacidad de generar propuestas de productos y servicios innovadores, adoptando modelos de negocios centrados en las necesidades de los clientes, adecuando los procesos de gestión que promuevan la implantación de iniciativas que impulsen el desarrollo cognitivo de la organización y sus colaboradores.

El desarrollo cognitivo de las empresas permite generar aprendizaje organizacional, para lo cual la arquitectura en su diseño debe crear las condiciones para que sus procesos estén en capacidad de dinamizar la producción de conocimiento. Es por consiguiente que el liderazgo de la empresa deba crear las condiciones para que se practiquen y se ejecuten los conocimientos generados y aprendidos. Ese es el rol correcto de alta dirección (Drucker, 2005).

Es por ello que la estructura y diseño, como componente sustantivo de la arquitectura, deben concebirse para responder con sentido de urgencia a las oportunidades del entorno. Cuando la estructura y el diseño de la organización son inteligentes, las organizaciones adoptan una nueva arquitectura organizacional, que en su accionar evidencia las siguientes características:

1. Son adaptativas a los procesos de cambios.

2. Sus procesos están centrados en el cliente.

3. Sus capacidades potencializan el logro de las estrategias.

4. Las estructuras formales e informales coexisten para el logro de las metas.

5. Los sistemas de control facilitan de manera selectiva la toma de decisión correctivas y medidas de mejoramiento continuo.

6. Los sistemas de incentivos y de control están alineados y fomentan la motivación del empleado.

7. La cultura y el clima organizacional juegan un papel importante para incrementar el rendimiento organizacional.

Como se puede apreciar, las exigencias por adoptar este proceso de cambio plantean como reto que la nueva arquitectura pueda crear las condiciones para que los equipos de trabajos sean eficaces y eficientes en su compromiso para alcanzar las metas de negocios establecidas. 
Parece oportuno avanzar en un modelo de interdependencia estratégica y estructural, pues el COVID ha afectado o afectará las arquitecturas organizacionales, lo cual significa generar una ruptura en los sistemas sociotécnicos de las organizaciones.

\section{Referencias}

Bergoglio, J. (2004). Anatomia de la empresa: una teoría general de las organizaciones sociales. México: Limusa, Noriega Editores.

Bierly, P. E; Spender, J. C. (1995). "Culture and high reliability organizations: The case of the nuclear submarine", Journal of Management, 21(4), 639-656.

Blau, M., y Scott, R.W. (2003). Formal organizations: a comparative approach. San Francisco: Chandler Publishing Company Stanford University Press, Business \& Economics.

Chiavenato I., (2006). Introducción a la teoría general de la administración. México: Editorial Mc Graw-Hill.

Chiavenato, I., (2009). Comportamiento organizacional: la dinámica del éxito en las organizaciones. (2a. ed.). México: Mc Graw-Hill.

Comisión Económica para América Latina y el Caribe. (2020). Observatorio COVID-19 en América Latina y el Caribe Impacto económico y social. Recuperado de https://www.cepal.org/es/temas/ covid-19 26/6/2020

Cummings, T. y Worley, C. (2007). Desarrollo organizacional y cambio. (8va. ed.). México: CENGAGE Learning.

Dávila L., J. C. (2013) Capacidades organizacionales: dinámicas por naturaleza. Cuadernos de Administración, 26(47): 11-33. Bogotá: Pontificia Universidad Javeriana Recuperado de https://www. redalyc.org/pdf/205/20531182002.pdf

Donaldson, L. (2001). The Contingency Theory of Organizations Australian School of Business. University of New South Wales, Sydney, Australia: SAGE Publishing.

Donnely J, Gibson J., e Ivancevich, J. (1995). Dirección y Administración de Empresas (8va. ed.). Willmengton: Addison-Wesley Iberoamericana 
Drucker, P. (2005). Administración para el futuro. La década de los noventa y más allá. España: Parramón Ediciones.

Etzioni, A. (1994). Organizaciones modernas. México: Unión Tipográfica Editorial Hispano-Americana.

Fukuyama, F. (2010). Confianza: las virtudes sociales y la creación de prosperidad. Madrid: Comercial Atheneum.

Gnyawali, D.y Madhavan, R. (2001). "Cooperative Network and Competitive Dynamics: A Structural Embeddedness Perspective". Academy of Management Review, 26(3): 431-445.

Grant, R. (1991). The resource-based theory of competitive advantege. Implications for strategy. California Management Review, 33(3): 114-135.

Guerras, L.A., De La Fuente, J., García-Tenorio, J., Guerras, L., Y Hernán Gomez, J. (2003). Diseño Organizativo de la Empresa. Madrid: Editorial Civitas, S.A.

Hall, R. (1996). Organizaciones: estructuras, procesos, resultados. México: Prentice.

Hanzon, D. K. (1993). Informal Networks: The Company Behind the rt. Massachusetts: Harvard Business Review.

Hill, C., Jones, G., y Schilling, M. (2015). Administración estratégica: teoría y casos. Un enfoque integral (11va ed.). México, D.F.: Cengage Learning Editores.

Kast, F. y Rosenzwing, J. (1995). Administración en las organizaciones. Enfoque de Sistema de Contingencia. México: Mc Graw-Hill.

Kopelman, R., Brief, A., y Guzzo, R. A. (1990). The role of climate and culture in productivity. Organizational climate and culture: 282-318.

Lawrence, P. y Lorsch, J. (1973). Organización y Ambiente. Barcelona: Editorial Labor.

Mintzberg, H, (1993). La estructura de las organizaciones. Capítulo 1, (pp. 25-26). Barcelona: Ariel.

Mintzberg, H. (2013). Simply Managing: What Managers Do — and Can Do Better. Berrett-Koehler Publishers,

Navas, J. y Guerras, N. (2001). Administración estratégica. Madrid: Civitas. 
Organización Internacional del Trabajo. (2020, 4 de julio). Claves para un teletrabajo eficaz durante la pandemia del COVID-19. Recuperado de https://www.ilo.org/global/about-the-ilo/newsroom/ news/WCMS_740038/lang--es/index.htm

Peterson, D. (2011). Partner selection for corporate social responsibility efforts: The case of choosing NGO partners using transaction cost analysis and resource dependency. Interdisciplinary Journal of Contemporary Research in Business, 2(11): 12-22.

Pfeffer, J. (1987). Organizaciones y teoría de la organización. Argentina: Editorial el Ateneo.

Rivas, T. (2001). ¿Nuevas Teorías de la Organización? Investigación Administrativa: 101-114.

Robbins, S. (2010). Comportamiento Organizacional, Conceptos, Controversias y Aplicaciones. (8va ed.). México: Pearson, Prentice Hall.

Schein, E. (1988). La cultura empresarial y el liderazgo. Una visión dinámica. España: Plaza \& Janes Editores.

Stoner, J., Freeman, R. E., Gilbert, D. R. (1995). Administración (5ta ed.). México: Prentice Hall.

Suescun, R. (2020, 16 de mayo). Viaje a la dimensión desconocida: opciones fundamentales de política y riesgos fiscales en tiempos del COVID-19 [Blogspot]. Recuperado de https://blogs.iadb. $\mathrm{org} /$ gestion-fiscal/es/viaje-dimension-desconocida-opciones-fundamentales-politica-riesgos-fiscales-covid-19/?j=406986\&sfmc_ sub $=10111099 \& \mathrm{l}=295 \_$HTML\&u=8156992\& $\& \mathrm{mid}=$ $100028582 \& \mathrm{jb}=36 \#$ ftn2

Trist, E. (1981). The socio. Technical perspective. The evolution of social technical systems as a conceptual framework and as an action research program. In a. H VAV DE VEN.

Wernerfelt, B. (1989). From Critical Resources to Corporate Strategy. Journal of General Management, 14: 4-12.

Zirilli, A. (1978). Fundamentos de organización y dirección general. España: Ediciones Deusto S.A. 
